\title{
Generalized Mutual Synchronization between Two Controlled Interdependent Networks
}

\author{
Quan Xu, ${ }^{1,2}$ Shengxian Zhuang, ${ }^{1}$ Dan $\mathrm{Hu}^{2}{ }^{2}$ Yingfeng Zeng, ${ }^{1}$ and Jian Xiao \\ ${ }^{1}$ Key Laboratory of Magnetic Suspension Technology and Maglev Vehicle, School of Electrical Engineering, \\ Southwest Jiaotong University, Chengdu 610031, China \\ ${ }^{2}$ School of Mechanical Engineering and Automation, Xihua University, Chengdu 610039, China
}

Correspondence should be addressed to Quan Xu; quanxnjd@sina.com

Received 13 January 2014; Accepted 6 February 2014; Published 11 March 2014

Academic Editor: Zhengguang Wu

Copyright (C) 2014 Quan Xu et al. This is an open access article distributed under the Creative Commons Attribution License, which permits unrestricted use, distribution, and reproduction in any medium, provided the original work is properly cited.

\begin{abstract}
This paper mainly focuses on the generalized mutual synchronization between two controlled interdependent networks. First, we propose the general model of controlled interdependent networks $A$ and $B$ with time-varying internetwork delays coupling. Then, by constructing Lyapunov functions and utilizing adaptive control technique, some sufficient conditions are established to ensure that the mutual synchronization errors between the state variables of networks $A$ and $B$ can asymptotically converge to zero. Finally, two numerical examples are given to illustrate the effectiveness of the theoretical results and to explore potential application in future smart grid. The simulation results also show how interdependent topologies and internetwork coupling delays influence the mutual synchronizability, which help to design interdependent networks with optimal mutual synchronizability.
\end{abstract}

\section{Introduction}

In recent years, extensive efforts have been devoted to understanding the properties of complex networks [1-5]. Particularly, as one of the most interesting and significant collective behaviors in real world, synchronization in complex dynamical networks has received increasing interest owing to its many potential applications in nature, socioeconomic systems, or engineering [6]. In the existing literature, it has been recognized that the network topology plays a significant role in synchronizability of diffusively coupled complex networks $[7,8]$. Also, by using some effective control schemes, a variety of synchronization phenomena have been discovered in various complex networks (see [918] and relevant references therein). However, the studies mentioned above focused almost exclusively on the inner synchronization inside a single, noninteracting network.

Li et al. [19] studied the outer synchronization (in this paper, we call it mutual synchronization to be defined in Section 2) referring to the synchronization between two or more networks. However, to the best of our knowledge, it can be realized mainly by the open-plus-closed-loop method $[19,20]$ or based on the drive-response concept [21-27] considering only the intranetwork coupling of network itself. Zheng et al. [28] and Wu et al. [29] further studied the outer synchronization between two complex networks considering two kinds of internetwork coupling, but nevertheless, they both still derived the synchronization criteria based on driveresponse concept and did not place the outer synchronization in the context of interdependent networks.

It is well known that many real-world network systems do interact with and depend on each other; for instance, various infrastructures such as transportation, water supply, fuel, and power stations are coupled together; realistic neuronal networks have a clustered structure and they can be viewed as interdependent networks; the epidemic can spread between the coupled networks of the infection layer and the prevention layer; dealing with secure information and cryptography, one can couple two systems to achieve the mutual synchronization, and so forth. Recently, Buldyrev et al. [30] studied the interdependent networks by presenting future smart grid as a real-life example, where the electrical power grid depends on the information network for control and the information network depends on the electrical power grid for their electricity supply. Then, Mei et al. [31] emphasized that it was urgent to research interdependent networks 
theory for smart grid. Also, Brummitt et al. [32] demonstrated how interdependence affected cascades of load using a multiple branching process approximation. In a word, efforts have been directed to the cascading failures and robustness of interdependent networks [33-37]. In general, it has been recognized that interdependent topologies, especially interlinking strategy and internetwork coupling strength, play a vital role in cascading behaviors and robustness of interdependent networks. Analogously, this motivates us to attempt to explore the effects of interdependent topologies on the mutual synchronization between two interdependent networks.

Quite recently, Um et al. [38] placed synchronization behavior in the context of interdependent networks, where the one-dimensional regular network is mutually coupled to the WS small-world network. Based on the mean-field analytic approach, it has been revealed that the internetwork coupling and the intranetwork coupling play different roles in the synchronizability of the WS network. However, it is still limited to inner synchronization in one of the two interdependent networks and hence it is necessary and significant to study the mutual synchronization between two controlled interdependent networks.

The major contributions of our work are as follows. First, we propose the general model of two controlled interdependent networks $A$ and $B$, which take into account not only the intranetwork coupling, but also the time-varying internetwork delays coupling. Second, we place the synchronization in the context of two controlled interdependent networks and study the generalized mutual synchronization of the proposed model. Third, in the numerical examples, to explore the potential application in smart grid, we couple the NW small-world network described by chaotic power system nodes and the scale-free network described by Lorenz chaotic systems following two interdependent interlinking strategies, respectively. Finally, we verify the influences of intranetwork and internetwork coupling and internetwork delays on the controlled mutual synchronizability, which can help to design the optimal interdependent networks.

The remaining part of this paper is organized as follows. Section 2 introduces some useful mathematical preliminaries and proposes the general model of two controlled interdependent networks. The generalized mutual synchronization is investigated and the main theoretical results of this paper are given in Section 3. In Section 4, two numerical examples are provided to explore the potential application in smart grid and to illustrate the correctness and effectiveness of the theoretical results. Finally, some conclusions and further work are given in Section 5.

\section{Preliminaries and Model Presentation}

2.1. Notations. The standard mathematical notations will be utilized throughout this paper. Let $\mathbb{R} \in(-\infty,+\infty), \mathbb{R}^{m}$ be the $m$-dimensional Euclidean space and let $\mathbb{R}^{m \times n}$ be the space of $m \times n$ real matrices; $\mathbf{I}_{n} \in \mathbb{R}^{n \times n}$ denotes the $n$-dimensional identity matrix; we use $\mathbf{A}^{T}$ or $\mathbf{x}^{T}$ to denote the transpose of the matrix $\mathbf{A}$ or the vector $\mathbf{x}$, respectively; $\lambda_{\max }$ is the maximum eigenvalue of corresponding real symmetric matrix;
$\|\mathbf{x}\|=\sqrt{\mathbf{x}^{T} \mathbf{x}}$ stand for the 2 -norm of the vector $\mathbf{x} ; \bigotimes$ presents the Kronecker product of two matrices.

2.2. Model of Two Controlled Interdependent Networks. For simplicity and without loss generality, we consider the following model of two controlled interdependent networks (1) and (2) (we call networks $A$ and $B$, respectively, in this paper) consisting of $N$ identical nodes with time-varying internetwork delays coupling. The dynamical equations for the model of controlled interdependent networks $A$ and $B$ can be given by

$$
\begin{aligned}
\dot{\mathbf{x}}_{i}(t)= & f\left(\mathbf{x}_{i}(t)\right)+a^{i} \sum_{j=1}^{N} a_{i j} \boldsymbol{\Gamma}_{1} \mathbf{x}_{j}(t)+c^{i} \sum_{j}^{N} c_{i j} \boldsymbol{\Gamma}_{3} \mathbf{y}_{j}\left(t-\tau_{1}(t)\right) \\
\dot{\mathbf{y}}_{i}(t)= & g\left(\mathbf{y}_{i}(t)\right)+b^{i} \sum_{j=1}^{N} b_{i j} \boldsymbol{\Gamma}_{2} \mathbf{y}_{j}(t) \\
& +d^{i} \sum_{j}^{N} d_{i j} \boldsymbol{\Gamma}_{4} \mathbf{x}_{j}\left(t-\tau_{2}(t)\right)+\mathbf{u}_{i}(t), \quad i=1,2, \ldots N,
\end{aligned}
$$

where $\mathbf{x}_{i}(t)=\left(x_{i 1}(t), x_{i 2}(t), \ldots x_{i m}(t)\right)^{T} \in \mathbb{R}^{m}\left(\mathbf{y}_{i}(t)=\right.$ $\left.\left(y_{i 1}(t), y_{i 2}(t), \ldots y_{i n}(t)\right)^{T} \in \mathbb{R}^{n}\right)$ is the state variable of the $i$ th node in network $A(B)$ at time $t ; f: \mathbb{R}^{+} \times \mathbb{R}^{m} \rightarrow$ $\mathbb{R}^{m}\left(g: \mathbb{R}^{+} \times \mathbb{R}^{n} \rightarrow \mathbb{R}^{n}\right)$ is a smooth vector function; $\mathbf{A}=\left(a_{i j}\right)_{N \times N}\left(\mathbf{B}=\left(b_{i j}\right)_{N \times N}\right)$ stands for the intranetwork coupling matrix describing the topological structure of the network $A(B)$; namely, if there is a connection from node $i$ to node $j$ in network $A(B)$, then $a_{i j}\left(b_{i j}\right)=1$; otherwise, $a_{i j}\left(b_{i j}\right)=0$; however, $\mathbf{C}=\left(c_{i j}\right)_{N \times N}\left(\right.$ or $\left.\mathbf{D}=\left(d_{i j}\right)_{N \times N}\right)$ is the internetwork coupling matrix representing the direct interaction from $i$ in network $A$ to $j$ in network $B$ (or from $i$ in network $B$ to $j$ in network $A$ ); that is, if there exists a connection from $i$ in network $A$ to $j$ in network $B$ (or from $i$ in network $B$ to $j$ in network $A$ ), then $c_{i j}\left(d_{i j}\right)=1$; otherwise, $c_{i j}\left(d_{i j}\right)=0 ; a^{i}\left(b^{i}\right)$ and $c^{i}\left(d^{i}\right)$ are the intranetwork and internetwork coupling strength for node $i$, respectively; $\boldsymbol{\Gamma}_{1} \in R^{m \times m}\left(\boldsymbol{\Gamma}_{2} \in \mathbb{R}^{n \times n}, \boldsymbol{\Gamma}_{3} \in \mathbb{R}^{m \times n}, \boldsymbol{\Gamma}_{4} \in \mathbb{R}^{n \times m}\right)$ is an inner coupling matrix describing the interactions between the coupled variables; $\tau_{1}(t), \tau_{2}(t)$ are the time-varying internetwork coupling delays between networks $A$ and $B$, respectively; $\mathbf{u}_{i}(t) \in R^{n}$ are the nonlinear controllers to be designed later for the mutual synchronization.

2.3. Mathematical Preliminaries. In order to obtain our theoretical results in Section 3, we introduce some necessary definitions, assumptions, and lemmas.

Definition 1. Let $\varphi_{i}(\cdot): \mathbb{R}^{m} \rightarrow \mathbb{R}^{n}(i=1,2, \ldots N)$ be the smooth vector functions. We define the generalized mutual synchronization errors as

$$
\mathbf{e}_{i}(t)=\mathbf{y}_{i}(t)-\varphi_{i}\left(\mathbf{x}_{i}(t)\right), \quad i=1,2, \ldots N .
$$

Thus, network $A$ is said to achieve generalized mutual synchronization with network $B$ successfully if

$$
\lim _{t \rightarrow \infty}\left\|\mathbf{e}_{i}(t)\right\|=0, \quad i=1,2, \ldots N .
$$


Assumption 2. Suppose that the vector function $g(\cdot)$ is Lipschitz continuous, namely, for any $\mathbf{x} \in \mathbb{R}^{n}, \mathbf{y} \in \mathbb{R}^{n}$ and a constant $\mu>0$, the following inequality holds:

$$
\|g(\mathbf{y})-g(\mathbf{x})\| \leq \mu\|\mathbf{y}-\mathbf{x}\|
$$

Assumption 3. Suppose that the time-varying delays $\tau_{1}(t)$, $\tau_{2}(t)$ are continuous differentiable functions with $0 \leq$ $\tau_{1}(t), \tau_{2}(t) \leq h<\infty$ and $0 \leq \dot{\tau}_{1}(t) \leq \varepsilon_{1}<1$. Clearly, this assumption holds for constant $\tau_{1}(t), \tau_{2}(t)$.

Remark 4. Assumptions 2 and 3 are both general assumptions, which hold for a broad class of real-world chaotic systems, such as Lorenz system, Chua's oscillator, Chen system, and Lü system [28]. Hence, in the following sections, we always assume that both assumptions hold.

Lemma 5 (see [26]). If there are any vectors $\mathbf{x}, \mathbf{y} \in \mathbb{R}^{n}$, then the following inequality is true:

$$
\mathbf{x}^{T} \mathbf{y} \leq \frac{1}{2} \mathbf{x}^{T} \mathbf{x}+\frac{1}{2} \mathbf{y}^{T} \mathbf{y}
$$

\section{Generalized Mutual Synchronization Criteria}

In this section, by designing appropriate adaptive controllers, we can establish some sufficient conditions to insure the generalized mutual synchronization of the proposed general model in Section 2. Obviously, we can deduce some similar criteria for any simple or typical examples from this general model.

Combining (1) and (2) and (3), we can express error system of controlled interdependent networks $A$ and $B$ in terms of

$$
\begin{aligned}
& \dot{\mathbf{e}}_{i}(t)= \dot{\mathbf{y}}_{i}(t)-\mathbf{J} \dot{\mathbf{x}}_{i}(t) \\
&= g\left(\mathbf{y}_{i}(t)\right)-\mathbf{J} f\left(\mathbf{x}_{i}(t)\right)+b^{i} \sum_{j=1}^{N} b_{i j} \boldsymbol{\Gamma}_{2} \mathbf{e}_{j}(t) \\
&-c^{i} \mathbf{J} \sum_{j=1}^{N} c_{i j} \boldsymbol{\Gamma}_{3} \mathbf{e}_{j}\left(t-\tau_{1}(t)\right) \\
&+b^{i} \sum_{j=1}^{N} b_{i j} \boldsymbol{\Gamma}_{2} \varphi_{j}\left(\mathbf{x}_{j}(t)\right)-a^{i} \mathbf{J} \sum_{j=1}^{N} a_{i j} \boldsymbol{\Gamma}_{1} x_{j}(t) \\
&+d^{i} \sum_{j=1}^{N} d_{i j} \boldsymbol{\Gamma}_{4} \mathbf{x}_{j}\left(t-\tau_{2}(t)\right) \\
&-c^{i} \mathbf{J} \sum_{j=1}^{N} c_{i j} \boldsymbol{\Gamma}_{3} \varphi_{j}\left(\mathbf{x}_{j}\left(t-\tau_{1}(t)\right)\right)+\mathbf{u}_{i}(t), \\
& i=1,2, \ldots N,
\end{aligned}
$$

where $\mathbf{J}=D \varphi_{i}\left(\mathbf{x}_{i}\right)$ is the Jacobian matrix of the function $\varphi_{i}\left(\mathbf{x}_{i}\right)$.
Remark 6. From (7), one can find that adding appropriate controller to nodes is an alternative method to obtain mutual synchronization between two networks. In this paper, we thus mainly focus on the controlled mutual synchronization between two networks in the general context of two interdependent networks. Therefore, the intranetwork coupling matrices $\mathbf{A}$ and $\mathbf{B}$ and the internetwork coupling matrices $\mathbf{C}$ and $\mathbf{D}$ can be chosen arbitrarily, meaning that it is not necessary for assuming diffusivity, symmetry, or irreducibility of the matrices $\mathbf{A}, \mathbf{B}, \mathbf{C}$, and $\mathbf{D}$. In addition, the topology structure, node dynamics, and dimension of state vector of one network can be different from the other.

Remark 7. It is well known that the time delays commonly exist in node dynamics, intranetwork coupling, and internetwork coupling. However, we just consider the time-varying internetwork coupling delays regardless of the others to explore the effects of internetwork coupling behavior on the mutual synchronization. It is noted that many networks of interest, like the Kuramoto model, have nonlinear coupling functions. Similarly, for simplicity, we just consider the linear intranetwork and internetwork coupling.

Theorem 8. Suppose that Assumptions 2 and 3 hold and that the adaptive controllers (8) and the corresponding update laws (9) are added to the error system (7). Thus, generalized mutual synchronization between controlled interdependent networks $A$ and $B$ with time-varying internetwork delays coupling can be asymptotically realized. Consider

$$
\begin{aligned}
\mathbf{u}_{i}(t)= & \mathbf{J} f\left(\mathbf{x}_{i}(t)\right)-g\left(\varphi_{i}\left(\mathbf{x}_{i}(t)\right)\right)+a^{i} \mathbf{J} \sum_{j=1}^{N} a_{i j} \boldsymbol{\Gamma}_{1} \mathbf{x}_{j}(t) \\
& -b^{i} \sum_{j=1}^{N} b_{i j} \boldsymbol{\Gamma}_{2} \varphi_{j}\left(\mathbf{x}_{j}(t)\right) \\
& +c^{i} \mathbf{J} \sum_{j=1}^{N} c_{i j} \boldsymbol{\Gamma}_{3} \varphi_{j}\left(\mathbf{x}_{j}\left(t-\tau_{1}(t)\right)\right) \\
& -d^{i} \sum_{j=1}^{N} d_{i j} \boldsymbol{\Gamma}_{4} \mathbf{x}_{j}\left(t-\tau_{2}(t)\right)-K_{i} \mathbf{e}_{i}(t), \\
& \dot{K}_{i}=l_{i}\left\|\mathbf{e}_{i}(t)\right\|^{2}, \quad i=1,2, \ldots N,
\end{aligned}
$$

where $K_{i}$ are the time-varying feedback gain and $l_{i}$ are arbitrary positive constants.

Proof. Plugging (8) and (9) into (7), the error dynamical system can be rewritten as

$$
\begin{aligned}
\dot{\mathbf{e}}_{i}(t)= & g\left(\mathbf{y}_{i}(t)\right)-g\left(\varphi_{i}\left(\mathbf{x}_{i}(t)\right)\right)+b^{i} \sum_{j=1}^{N} b_{i j} \boldsymbol{\Gamma}_{2} \mathbf{e}_{j}(t) \\
& -c^{i} \mathbf{J} \sum_{j=1}^{N} c_{i j} \boldsymbol{\Gamma}_{3} \mathbf{e}_{j}\left(t-\tau_{1}(t)\right)-K_{i} \mathbf{e}_{i}(t), \quad i=1,2, \ldots N .
\end{aligned}
$$


Let $\mathbf{e}(t)=\left(\mathbf{e}_{1}^{T}(t), \mathbf{e}_{2}^{T}(t), \ldots, \mathbf{e}_{N}^{T}(t)\right)^{T} \in R^{n N}$, and construct a Lyapunov function as follows:

$$
\begin{aligned}
V(t)= & \frac{1}{2} \sum_{i=1}^{N} \mathbf{e}_{i}^{T}(t) \mathbf{e}_{i}(t)+\frac{1}{2} \sum_{j=1}^{N} \frac{1}{l_{i}}\left(K_{i}-\bar{K}\right)^{2} \\
& +\frac{1}{2} \sum_{i=1}^{N} \frac{1}{1-\varepsilon_{1}} \int_{t-\tau_{1}(t)}^{t} \mathbf{e}_{i}^{T}(\theta) \mathbf{e}_{i}(\theta) d \theta,
\end{aligned}
$$

where $\bar{K}$ is a positive constant large enough to be selected later. Obviously, $V(t)>0$ for all $\mathbf{e}(t) \neq 0$, meaning that $V(t)$ is positive definite. Calculating the derivative of (11) with respect to time along the solution of the error system (10), together with the updated laws (9), thus, we have

$$
\begin{aligned}
& \dot{V}(t)=\sum_{i=1}^{N} \mathbf{e}_{i}^{T}(t) \dot{\mathbf{e}}_{i}(t)+\sum_{i=1}^{N} \frac{1}{l_{i}}\left(K_{i}-\bar{K}\right) \dot{K}_{i} \\
& +\frac{1}{2\left(1-\varepsilon_{1}\right)} \sum_{i=1}^{N} \mathbf{e}_{i}^{T}(t) \mathbf{e}_{i}(t) \\
& -\frac{1-\dot{\tau}_{1}(t)}{2\left(1-\varepsilon_{1}\right)} \sum_{i=1}^{N} \mathbf{e}_{i}^{T}\left(t-\tau_{1}(t)\right) \mathbf{e}_{i}\left(t-\tau_{1}(t)\right) \\
& =\sum_{i=1}^{N} \mathbf{e}_{i}^{T}(t)\left[g\left(\mathbf{y}_{i}(t)\right)-g\left(\varphi_{i}\left(\mathbf{x}_{i}(t)\right)\right)-K_{i} \mathbf{e}_{i}(t)\right] \\
& +\sum_{i=1}^{N}\left(\frac{1}{2\left(1-\varepsilon_{1}\right)}+K_{i}-\bar{K}\right) \mathbf{e}_{i}^{T}(t) \mathbf{e}_{i}(t) \\
& +\sum_{i=1}^{N} \mathbf{e}_{i}^{T}(t)\left[\sum_{j=1}^{N} b^{i} b_{i j} \boldsymbol{\Gamma}_{2} \mathbf{e}_{j}(t)\right. \\
& \left.-\sum_{j=1}^{N} c^{i} c_{i j} \mathbf{J} \Gamma_{3} \mathbf{e}_{j}\left(t-\tau_{1}(t)\right)\right] \\
& -\frac{1-\dot{\tau}_{1}(t)}{2\left(1-\varepsilon_{1}\right)} \sum_{i=1}^{N} \mathbf{e}_{i}^{T}\left(t-\tau_{1}(t)\right) \mathbf{e}_{i}\left(t-\tau_{1}(t)\right) \\
& \leq \sum_{i=1}^{N} \mathbf{e}_{i}^{T}(t)\left(\mu+\frac{1}{2\left(1-\varepsilon_{1}\right)}-\bar{K}\right) \mathbf{e}_{i}(t) \\
& +\sum_{i=1}^{N} \sum_{j=1}^{N} \mathbf{e}_{i}^{T}(t) b^{i} b_{i j} \boldsymbol{\Gamma}_{2} \mathbf{e}_{j}(t) \\
& -\sum_{i=1}^{N} \sum_{j=1}^{N} \mathbf{e}_{i}^{T}(t) c^{i} c_{i j} \mathbf{J} \boldsymbol{\Gamma}_{3} \mathbf{e}_{j}\left(t-\tau_{1}(t)\right) \\
& -\frac{1-\dot{\tau}_{1}(t)}{2\left(1-\varepsilon_{1}\right)} \sum_{i=1}^{N} \mathbf{e}_{i}^{T}\left(t-\tau_{1}(t)\right) \mathbf{e}_{i}\left(t-\tau_{1}(t)\right) .
\end{aligned}
$$

Let $\mathbf{E}=\mathbf{G} \otimes \boldsymbol{\Gamma}_{2} ; \mathbf{G}=\left(b^{i} b_{i j}\right)_{N \times N} ; \mathbf{F}=\mathbf{H} \otimes\left(\mathbf{J} \boldsymbol{\Gamma}_{3}\right) ; \mathbf{H}=$ $\left(-c^{i} c_{i j}\right)_{N \times N}$, thus, we can get

$$
\begin{aligned}
\dot{V}(t) \leq & \left(\mu+\frac{1}{2\left(1-\varepsilon_{1}\right)}-\bar{K}\right) \mathbf{e}^{T}(t) \mathbf{e}(t) \\
& +\mathbf{e}^{T}(t) \frac{\mathbf{E}+\mathbf{E}^{T}}{2} \mathbf{e}(t)+\mathbf{e}^{T}(t) \mathbf{F e}\left(t-\tau_{1}(t)\right) \\
& -\frac{1-\dot{\tau}_{1}(t)}{2\left(1-\varepsilon_{1}\right)} \sum_{i=1}^{N} \mathbf{e}_{i}^{T}\left(t-\tau_{1}(t)\right) \mathbf{e}_{i}\left(t-\tau_{1}(t)\right) .
\end{aligned}
$$

From Assumption 3, we have

$$
\frac{1}{2}-\frac{1-\dot{\tau}_{1}(t)}{2\left(1-\varepsilon_{1}\right)} \leq 0
$$

From Lemma 5, we get

$$
\begin{aligned}
\mathbf{e}^{T}(t) \mathbf{F e}\left(t-\tau_{1}(t)\right) \leq & \frac{1}{2} \mathbf{e}^{T}(t) \mathbf{F F}^{T} \mathbf{e}(t) \\
& +\frac{1}{2} \mathbf{e}^{T}\left(t-\tau_{1}(t)\right) \mathbf{e}\left(t-\tau_{1}(t)\right) .
\end{aligned}
$$

Combining (14) and (15) and (13), we can further get

$$
\begin{aligned}
\dot{V}(t) \leq & \left(\mu+\frac{1}{2\left(1-\varepsilon_{1}\right)}-\bar{K}\right) \mathbf{e}^{T}(t) \mathbf{e}(t) \\
& +\mathbf{e}^{T}(t) \frac{\mathbf{E}+\mathbf{E}^{T}}{2} \mathbf{e}(t)+\frac{1}{2} \mathbf{e}^{T}(t) \mathbf{F F}^{T} \mathbf{e}(t) \\
& +\frac{1}{2} \mathbf{e}^{T}\left(t-\tau_{1}(t)\right) \mathbf{e}\left(t-\tau_{1}(t)\right) \\
& -\frac{1-\tau_{1}(t)}{2\left(1-\varepsilon_{1}\right)} \frac{1}{2} \mathbf{e}^{T}\left(t-\tau_{1}(t)\right) \mathbf{e}\left(t-\tau_{1}(t)\right) \\
\leq & \left(\mu+\frac{1}{2\left(1-\varepsilon_{1}\right)}-\bar{K}\right) \mathbf{e}^{T}(t) \mathbf{e}(t) \\
& +\mathbf{e}^{T}(t) \frac{\mathbf{E}+\mathbf{E}^{T}}{2} \mathbf{e}(t)+\frac{1}{2} \mathbf{e}^{T}(t) \mathbf{F} \mathbf{F}^{T} \mathbf{e}(t) \\
\leq & \left(\mu+\frac{1}{2\left(1-\varepsilon_{1}\right)}-\bar{K}+\lambda_{\max }\left(\frac{\mathbf{E}+\mathbf{E}^{T}}{2}\right)\right. \\
& \left.+\lambda_{\max }\left(\mathbf{F F}^{T}\right)\right) \mathbf{e}^{T}(t) \mathbf{e}(t)
\end{aligned}
$$

If we take $\bar{K}$ as

$$
\bar{K} \geq \mu+\frac{1}{2\left(1-\varepsilon_{1}\right)}+\lambda_{\max }\left(\frac{\mathbf{E}+\mathbf{E}^{T}}{2}\right)+\lambda_{\max }\left(\mathbf{F F}^{T}\right)+1,
$$


then

$$
\dot{V}(t) \leq-\mathbf{e}^{T}(t) \mathbf{e}(t)=-\|\mathbf{e}(t)\|^{2} \leq 0 .
$$

Clearly, $V(t)$ is nonincreasing and every term of $V(t)$ is bounded. Thus, $\lim _{t \rightarrow \infty} V(t)$ tends to a nonnegative value. Since $K_{i}$ is bounded and increasing (see (8) and (9)), it must also asymptotically converge to a limit. By integrating (18) over 0 to $t$, we can get $\int_{0}^{t}\|\mathbf{e}(\theta)\|^{2} d \theta \leq$ $-\int_{0}^{t} \dot{V}(\theta) d \theta$. Thus, $\lim _{t \rightarrow \infty} \int_{0}^{t}\|\mathbf{e}(\theta)\|^{2} d \theta$ exists and is a nonnegative value. According to Cauchy Criterion, we can obtain $\lim _{t \rightarrow+\infty} \int_{t-\tau_{1}(t)}^{t} \mathbf{e}_{i}^{T}(\theta) \mathbf{e}_{i}(\theta) d \theta=0$. Therefore, from the definition of $V(t)$, we can conclude that $\lim _{t \rightarrow \infty}\|\mathbf{e}(t)\|^{2}$ converges to a limited nonnegative constant. Next, we would prove that $\lim _{t \rightarrow \infty}\|\mathbf{e}(t)\|^{2}=0$. If this is not true, then $\lim _{t \rightarrow+\infty}\|\mathbf{e}(t)\|^{2}=\epsilon(\epsilon>0)$ holds. Obviously, $\|\mathbf{e}(t)\|^{2}>\epsilon / 2$ holds true for $t \geq \delta>0$. From (18), we have

$$
\dot{V}(t) \leq-\|\mathbf{e}(t)\|^{2}<-\frac{\epsilon}{2} .
$$

Thus, by integrating (19) from $H$ to $\infty$, we can get

$$
\begin{aligned}
-V(\delta) & \leq V(+\infty)-V(\delta)=\int_{\delta}^{+\infty} \dot{V}(t) d t \\
& <-\int_{\delta}^{+\infty} \frac{\epsilon}{2} d t=-\infty .
\end{aligned}
$$

This is a contradiction, and hence $\lim _{t \rightarrow+\infty}\|\mathbf{e}(t)\|^{2}=0$; namely, $\lim _{t \rightarrow \infty}\left\|\mathbf{e}_{i}(t)\right\|=0, i=1,2, \ldots N$. Consequently, the generalized mutual synchronization between controlled interdependent networks $A$ and $B$ is asymptotically obtained by using the proposed adaptive controllers (8) and (9). This completes the proof of the Theorem 8 .

Remark 9. From the proof of the Theorem 8, we know that $V(t)$ is positive definite, $\dot{V}(t)$ is negative definite, and $\lim _{t \rightarrow \infty} \mathbf{e}_{i}(t)=0$. According to Lyapunov stability theory, we can also get that the synchronization state $\mathbf{e}_{i}(t)=0$ is asymptotically stable.

Remark 10. It is noted that (17) is just a sufficient condition, but not the necessary one for the mutual synchronization between controlled interdependent networks $A$ and $B$.

Based on Theorem 8, we can further obtain some similar synchronization criteria in the following two corollaries.

Corollary 11. Suppose that Assumptions 2 and 3 hold. If $m=n$ and $\varphi_{i}\left(\mathbf{x}_{i}(t)\right)=\lambda \mathbf{x}_{i}(t), \lambda \neq 0$, then projective mutual synchronization between controlled interdependent networks $A$ and $B$ with time-varying internetwork delays coupling can be asymptotically achieved under the following adaptive control schemes:

$$
\begin{aligned}
\mathbf{u}_{i}(t)= & \lambda f\left(\mathbf{x}_{i}(t)\right)-g\left(\lambda x_{i}(t)\right) \\
& +\sum_{j=1}^{N} \lambda\left(a^{i} a_{i j} \boldsymbol{\Gamma}_{1}-b^{i} b_{i j} \boldsymbol{\Gamma}_{2}\right) \mathbf{x}_{j}(t) \\
& +\sum_{j=1}^{N} \lambda^{2} c^{i} c_{i j} \boldsymbol{\Gamma}_{3} \mathbf{x}_{j}\left(t-\tau_{1}(t)\right) \\
& -\sum_{j=1}^{N} d^{i} d_{i j} \boldsymbol{\Gamma}_{4} \mathbf{x}_{j}\left(t-\tau_{2}(t)\right)-K_{i} \mathbf{e}_{i}(t), \\
\quad i=1,2, \ldots N, & \dot{K}_{i}=l_{i}\left\|\mathbf{e}_{i}(t)\right\|^{2},
\end{aligned}
$$

where $K_{i}, l_{i}$ have the same implications as those of Theorem 8 , respectively.

Corollary 12. Particularly, in Corollary 11, if $\lambda= \pm 1$, complete mutual synchronization (mutual antisynchronization) between controlled interdependent networks $A$ and $B$ with time-varying internetwork delays coupling can be asymptotically obtained by the adaptive controllers as follows:

$$
\begin{aligned}
\mathbf{u}_{i}(t)= & f\left(\mathbf{x}_{i}(t)\right)-g\left( \pm \mathbf{x}_{i}(t)\right) \\
& \pm \sum_{j=1}^{N}\left(a^{i} a_{i j} \boldsymbol{\Gamma}_{1}-b^{i} b_{i j} \boldsymbol{\Gamma}_{2}\right) \mathbf{x}_{j}(t) \\
& +\sum_{j=1}^{N} c^{i} c_{i j} \boldsymbol{\Gamma}_{3} \mathbf{x}_{j}\left(t-\tau_{1}(t)\right) \\
& -\sum_{j=1}^{N} d^{i} d_{i j} \boldsymbol{\Gamma}_{4} \mathbf{x}_{j}\left(t-\tau_{2}(t)\right)-K_{i} \mathbf{e}_{i}(t), \\
\quad i=1,2, \ldots N, & \dot{K}_{i}=l_{i}\left\|\mathbf{e}_{i}(t)\right\|^{2},
\end{aligned}
$$

where $K_{i}, l_{i}$ have the same implications as those of Theorem 8 , respectively.

Remark 13. It is quite natural that Theorem 8 and Corollaries 11 and 12 still hold for some simple cases, such as $\tau_{1}(t)=0$, $\tau_{2}(t)=0, \mathbf{A}=\mathbf{B}, \mathbf{C}=\mathbf{D}, \boldsymbol{\Gamma}_{1}=\boldsymbol{\Gamma}_{2}$, and $\boldsymbol{\Gamma}_{3}=\boldsymbol{\Gamma}_{4}$; hence, our model and synchronization methods are applicable to some of the two controlled interdependent networks similar to our model.

Remark 14. Plugging (3) into (10) and (1), we obtain (23) and (24), respectively:

$$
\begin{aligned}
\dot{\mathbf{e}}_{i}(t)= & g\left(\mathbf{e}_{i}(t)+\varphi_{i}\left(\mathbf{x}_{i}(t)\right)\right)-g\left(\varphi_{i}\left(\mathbf{x}_{i}(t)\right)\right) \\
& +\sum_{j=1}^{N} b^{i} b_{i j} \boldsymbol{\Gamma}_{2} \mathbf{e}_{j}(t)
\end{aligned}
$$




$$
\begin{gathered}
-\mathbf{J} \sum_{j=1}^{N} c^{i} c_{i j} \boldsymbol{\Gamma}_{3} \mathbf{e}_{j}\left(t-\tau_{1}(t)\right)-K_{i} \mathbf{e}_{i}(t), \\
i=1,2, \ldots N, \\
\dot{\mathbf{x}}_{i}(t)=f\left(\mathbf{x}_{i}(t)\right)+\sum_{j=1}^{N} a^{i} a_{i j} \boldsymbol{\Gamma}_{1} \mathbf{x}_{j}(t) \\
+\sum_{j}^{N} c^{i} c_{i j} \boldsymbol{\Gamma}_{3}\left(\mathbf{e}_{i}(t)+\varphi_{i}\left(\mathbf{x}_{i}(t)\right)\right), \\
i=1,2, \ldots N .
\end{gathered}
$$

Combining (23) and (24), we find that the values of $e_{i}(t)$ are irrelevant to $\tau_{2}(t), d_{i j}$, and $d^{i}$ under the action of the proposed adaptive controllers (8) and (9). Thus, in the following sections, it is reasonable not to consider the effects of $\tau_{2}(t)$, $d_{i j}$, and $d^{i}$ on the mutual synchronization between controlled interdependent networks $A$ and $B$.

\section{Numerical Simulations and Results}

In this section, two numerical examples and their simulations are given to illustrate the correctness and effectiveness of the theoretical results obtained in the previous sections and to identify the factors that influence the mutual synchronizability.

To measure the speed and performance of mutual synchronization process, we define

$$
\|\mathbf{e}(t)\|=\sqrt{\sum_{i=1}^{N} \sum_{j=1}^{n} e_{i j}(t)^{2}}
$$

Actually, $\|\mathbf{e}(t)\|$ is the 2-norm of the synchronization error $\mathbf{e}(t), 0<t<+\infty$. Thus, the values of $\|\mathbf{e}(t)\|$ in the initial stage and at the end of simulations imply the mutual synchronization speed and performance, respectively. It should be particularly noted that, in all of the following simulations, the main figures and insets describe the values of $\|\mathbf{e}(t)\|$ during $0 \leq t<5$ and at the end of simulations $(t=5)$, respectively.

Next, to explore the potential application of mutual synchronization in smart grid, we construct network $A$ as NWsmall-world network $(N=50, k=3, P=0.3)$ consisting of identical chaotic power system nodes and network $B$ as scale-free network $\left(N=50, m=m_{0}=3\right)$ described by Lorenz chaotic systems. The nonlinear function $f\left(\mathbf{x}_{i}(t)\right)$ corresponding to chaotic power system nodes [39] is described by

$$
f\left(\mathbf{x}_{i}(t)\right)=\left(\begin{array}{c}
x_{i 2}(t) \\
-a_{1} \sin \left(x_{i 1}(t)\right)-b_{1} x_{i 2}(t)+c_{1}+F \cos \left(d_{1} t\right)
\end{array}\right) .
$$

When taking $a_{1}=1, b_{1}=0.02, c_{1}=0.2, d_{1}=1$, and $F=$ 0.296 , the above power system nodes are hyperchaotic. The nonlinear function $g\left(y_{i}(t)\right)$ involving the Lorenz systems [26] is represented by

$$
g\left(\mathbf{y}_{i}(t)\right)=\left(\begin{array}{c}
a_{2}\left(y_{i 2}(t)-y_{i 1}(t)\right) \\
b_{2} y_{i 1}(t)-y_{i 2}(t)-y_{i 1}(t) y_{i 3}(t) \\
-c_{2} y_{i 1}(t)+y_{i 1}(t) y_{i 2}(t)
\end{array}\right) .
$$

When taking $a_{2}=10, b_{2}=28$, and $c_{2}=8 / 3$, the Lorenz systems are chaotic. As is known to all, the chaotic systems are bounded, thus, $g\left(\mathbf{y}_{i}(t)\right)$ satisfies Assumption 2. In the both examples, we arbitrarily select the generalized mapping functions as $\varphi_{i}\left(\mathbf{x}_{i}(t)\right)=\left(x_{i 1}(t), x_{i 2}(t), x_{i 1}(t)+x_{i 2}(t)\right)^{T}$, meaning that $J=\left(\begin{array}{ll}1 & 0 \\ 0 & 1 \\ 1 & 1\end{array}\right)$; then, we set $l_{i}=1, \Gamma_{1}=\mathbf{I}_{2}, \Gamma_{2}=\mathbf{I}_{3}$, $\Gamma_{3}=\left(\begin{array}{lll}1 & 0 & 0 \\ 0 & 1 & 0\end{array}\right), \boldsymbol{\Gamma}_{4}=\left(\begin{array}{ll}1 & 0 \\ 0 & 1 \\ 0 & 0\end{array}\right)$; next, the initial values $\mathbf{x}_{i}(0)$ and $\mathbf{y}_{i}(0)$ can be chosen randomly in $(-1,1)$ and $K_{i}(0)$ in $(0,1)$; in addition, the internetwork delays will be selected according to the Assumption 3.

For simplicity and for comparing, we further assume that the internetwork coupling links are bidirectional and the coupling strength of each node is equal; that is, $a^{i}=a$, $b^{i}=b, c^{i}=c$, and $d^{i}=d$. From Remark 14, we know that the time evolutions of $\mathbf{e}_{i}(t)$ are not relevant to $\tau_{2}(t), d_{i j}$, and $d^{i}$; thus, it is also reasonable to assume $c=d=s$, $\tau_{1}(t)=\tau_{2}(t)=\tau(t)$ to simulate the influences of internetwork coupling strength and delays on the mutual synchronizability. Here, we employ the following two interlinking strategies to produce the interdependency matrices $\mathbf{C}$ and $\mathbf{D}$ in the two examples respectively.

(i) One-to-one support dependence interlinking strategy [30] (strategy I for short): node $A_{i}$ in network $A$ only depends on node $B_{i}$ in network $B$ and vice versa.

(ii) Multiple support dependence interlinking strategy [37] (strategy II for short): node in network $A$ may randomly depend on more than one node in network $B$ and vice versa.

Example 15. In this example, we generate the interdependency matrices $\mathbf{C}$ and $\mathbf{D}$ following the strategy I and design the adaptive controllers according to Theorem 8. When $a=$ $b=s=1, \tau(t)=0.5$, the mutual synchronization errors $\mathbf{e}_{i}(t)$ are depicted in Figure 1, which shows that controlled interdependent networks $A$ and $B$ can easily achieve the generalized mutual synchronization using the designed controllers. Next, we further simulate the influences of internetwork delays and intranetwork and internetwork coupling strength on the mutual synchronizability between the networks $A$ and $B$. We fix $a=b=s=1$ and only change the internetwork coupling delays $\tau(t)$; thus, the values of $\|e(t)\|$ for the networks $A$ and $B$ with different $\tau(t)$ are plotted in Figure 2. Similarly, Figures 3,4 , and 5 show the curves of $\|\mathbf{e}(t)\|$ for the networks $A$ and $B$ with fixed parameters $b=s=1, \tau(t)=0.5$ and different intranetwork coupling strength $a$, with $a=s=1, \tau(t)=0.5$ and different intranetwork coupling strength $b$, with $a=b=$ $1, \tau(t)=0.5$ and different internetwork coupling strength $s$, respectively. 

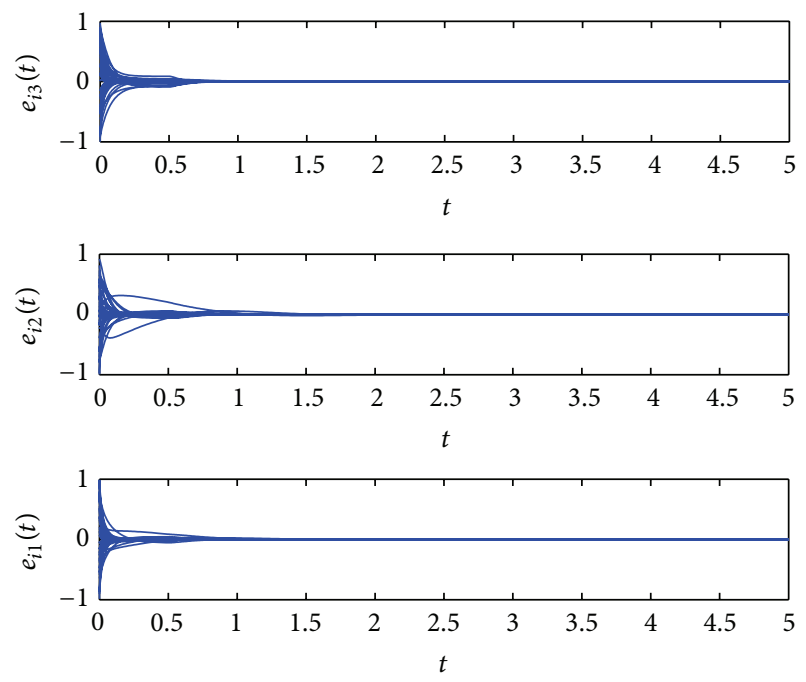

FIgURE 1: The mutual synchronization errors $\mathbf{e}_{i}(t)$ between the networks $A$ and $B$ interlinked following strategy I with $a=b=s=1$, $\tau(t)=0.5$.

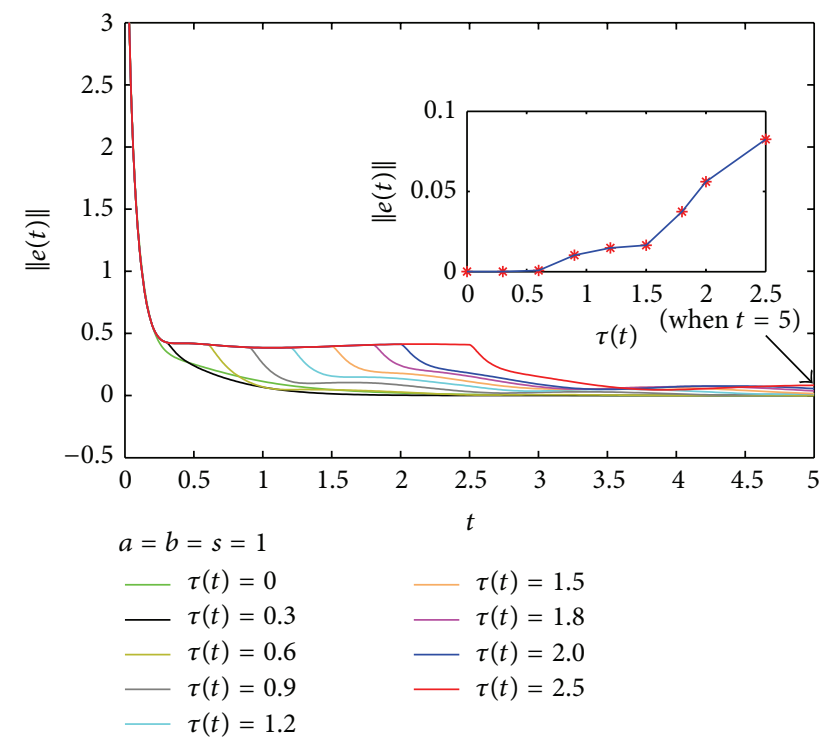

Figure 2: The curves of $\|\mathbf{e}(t)\|$ for the networks $A$ and $B$ interlinked following strategy I with $a=b=s=1$ and different internetwork delays $\tau(t)$.

Example 16. In this example, we produce the interdependency matrices $\mathbf{C}$ and $\mathbf{D}$ following the strategy II. To measure the effect of the number of interlinking edges on the mutual synchronizability, we define $\langle k\rangle$ as the average number of interlinking edges for each node in network $A$ and the same to network $B$. We conduct similar simulations as those in Example 15. First, we set $a=b=s=1,\langle k\rangle=$ 3, $\tau(t)=e^{t} /\left(1+e^{t}\right)$; thus, the time evolutions of the synchronization errors $\mathbf{e}_{i}(t)$ are depicted in Figure 6, which shows that interdependent networks $A$ and $B$ can achieve the generalized mutual synchronization successfully. Then, Figures 7, 8, 9, and 10, respectively, display the curves of $\|\mathbf{e}(t)\|$ for the networks $A$ and $B$ with fixed parameters $a=b=$

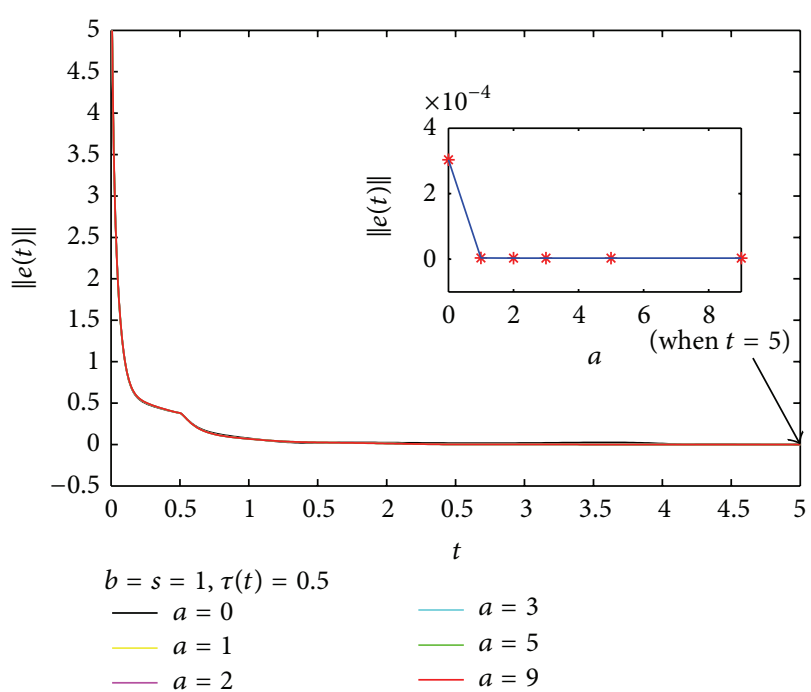

FIgURE 3: The curves of $\|\mathbf{e}(t)\|$ for the networks $A$ and $B$ interlinked following strategy I with $b=s=1, \tau(t)=0.5$ and different intranetwork strength $a$.

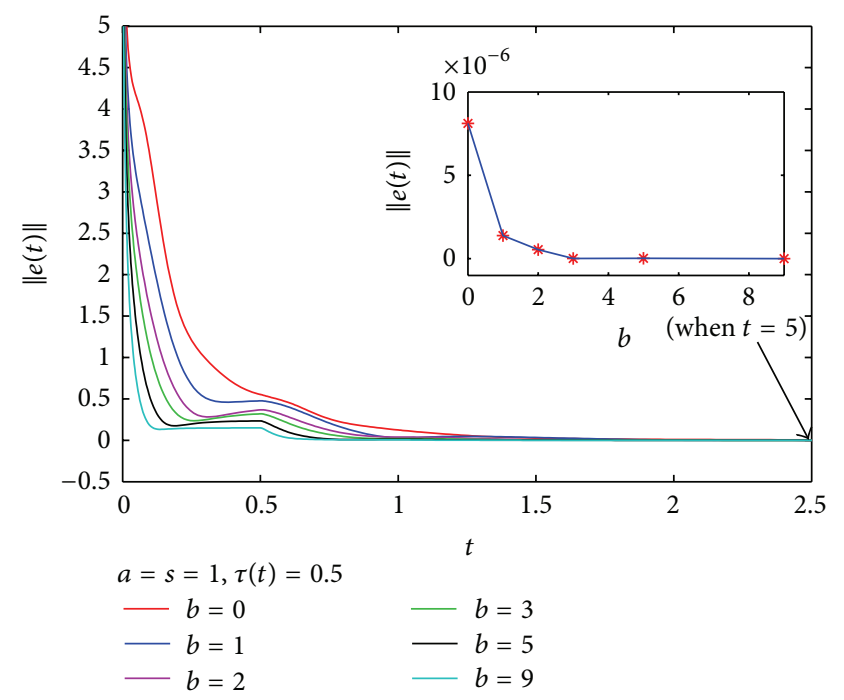

Figure 4: The curves of $\|\mathbf{e}(t)\|$ for the networks $A$ and $B$ interlinked following strategy I with $a=s=1, \tau(t)=0.5$ and different intranetwork strength $b$.

$s=1,\langle k\rangle=3$ and different internetwork delays $\tau(t)$, with $b=s=1,\langle k\rangle=3$, and $\tau(t)=e^{t} /\left(1+e^{t}\right)$ and different intranetwork coupling strength $a$, with $a=s=1,\langle k\rangle=3$, and $\tau(t)=e^{t} /\left(1+e^{t}\right)$ and different intranetwork coupling strength $b$, with $a=b=1,\langle k\rangle=3$, and $\tau(t)=e^{t} /\left(1+e^{t}\right)$ and different internetwork coupling strength $s$. Finally, we fix the parameters $a=b=s=1, \tau(t)=e^{t} /\left(1+e^{t}\right)$; thus, the curves of $\|\mathbf{e}(t)\|$ for the networks $A$ and $B$ with different $\langle k\rangle$ are shown in Figure 11.

From the numerical results, both examples yield coincident tendency as follows, which further affirms our theoretical results. It is observed that the intranetwork coupling $a$ has 


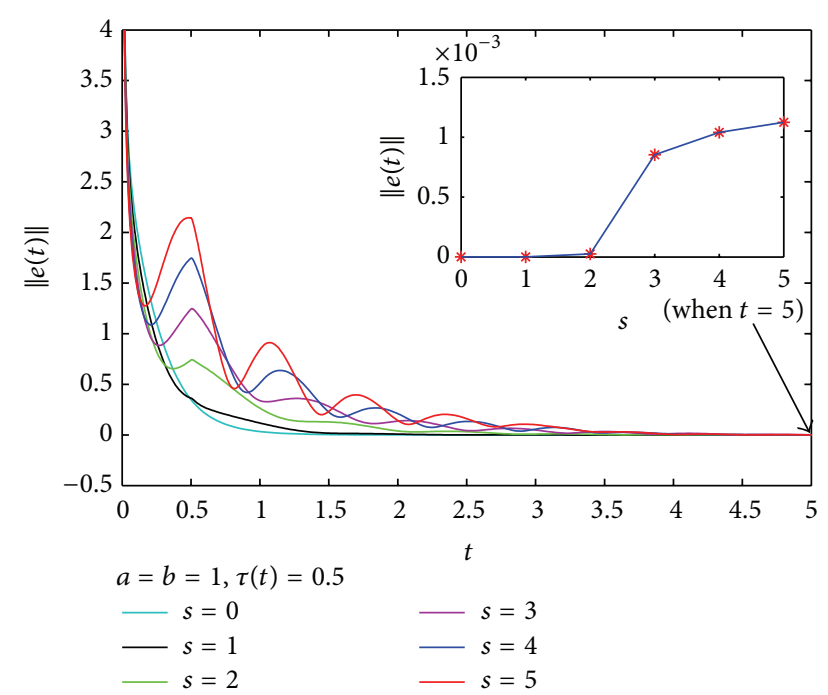

Figure 5: The curves of $\|\mathbf{e}(t)\|$ for the networks $A$ and $B$ interlinked following strategy I with $a=b=1, \tau(t)=0.5$ and different internetwork strength $s$.
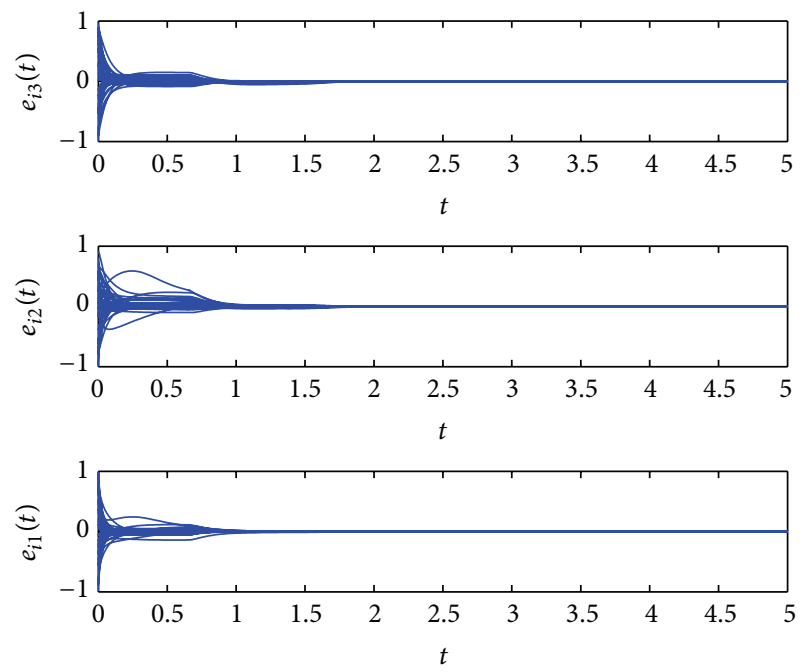

FIGURE 6: The mutual synchronization errors $\mathbf{e}_{i}(t)$ between interdependent networks $A$ and $B$ interlinked following strategy II with $a=b=s=1,\langle k\rangle=3$, and $\tau(t)=e^{t} /\left(1+e^{t}\right)$.

little influence on mutual synchronization process (shown in Figures 3 and 8 and their insets), and the stronger intranetwork coupling $b$ enhances the mutual synchronizability (shown in Figures 4 and 9 and their insets), while the stronger internetwork coupling worsen the mutual synchronizability (shown in Figures 5 and 10 and their insets). It is also found that the values of $\|\mathbf{e}(t)\|$ both in initial stage and at the end of simulations are increased as the internetwork coupling delay $\tau(t)$ is increased (shown in Figures 2 and 7 and their insets, resp.). In addition, Figure 11 implies that, to some extent, increase of $\langle k\rangle$ is equivalent to the increase of internetwork coupling strength $s$.

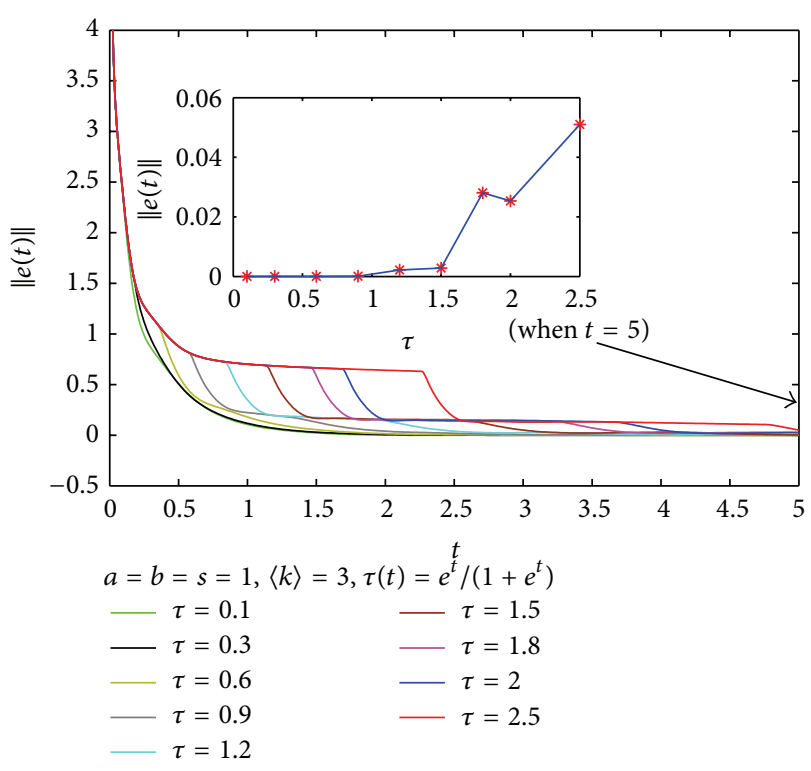

FIgURE 7: The curves of $\|\mathbf{e}(t)\|$ for the networks $A$ and $B$ interlinked following strategy II with $a=b=s=1,\langle k\rangle=3$ and different internetwork delays $\tau(t)$.

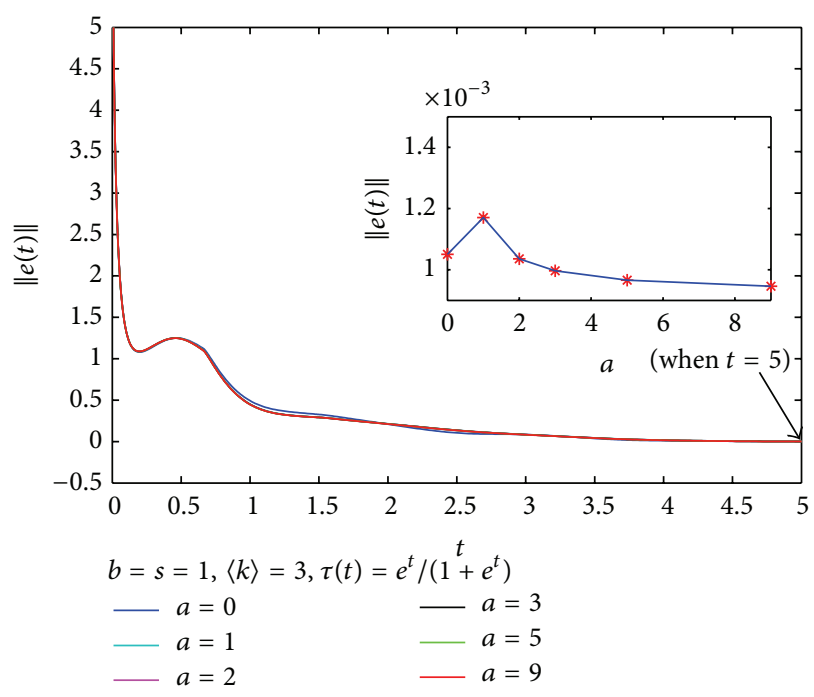

FIGURE 8: The curves of $\|\mathbf{e}(t)\|$ for the networks $A$ and $B$ interlinked following strategy II with $b=s=1,\langle k\rangle=3$, and $\tau(t)=e^{t} /\left(1+e^{t}\right)$ and different intranetwork strength $a$.

\section{Conclusions and Future Work}

In this paper, we extend previous research on the outer synchronization between two complex networks to our work on generalized mutual synchronization between two controlled interdependent networks by considering the time-varying internetwork delays coupling. Our model and relevant results are general and can be easily extended to other interdependent networks because there are not any constraints imposed on the intranetwork and internetwork coupling configuration matrices. Based on Lyapunov theory and corresponding mathematical techniques, some sufficient criteria have been 


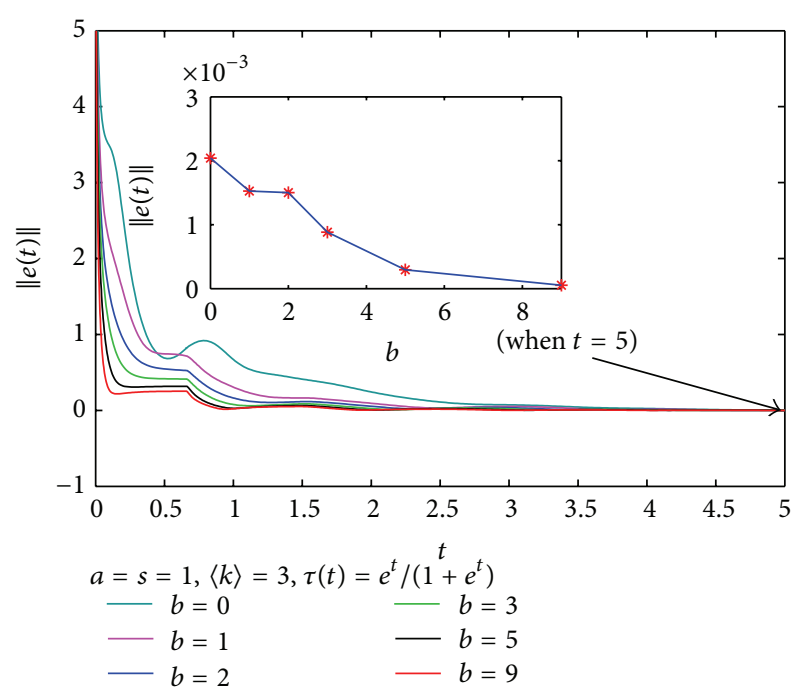

FIGURE 9: The curves of $\|\mathbf{e}(t)\|$ for the networks $A$ and $B$ interlinked following strategy II with $a=s=1,\langle k\rangle=3$, and $\tau(t)=e^{t} /\left(1+e^{t}\right)$ and different intranetwork strength $b$.

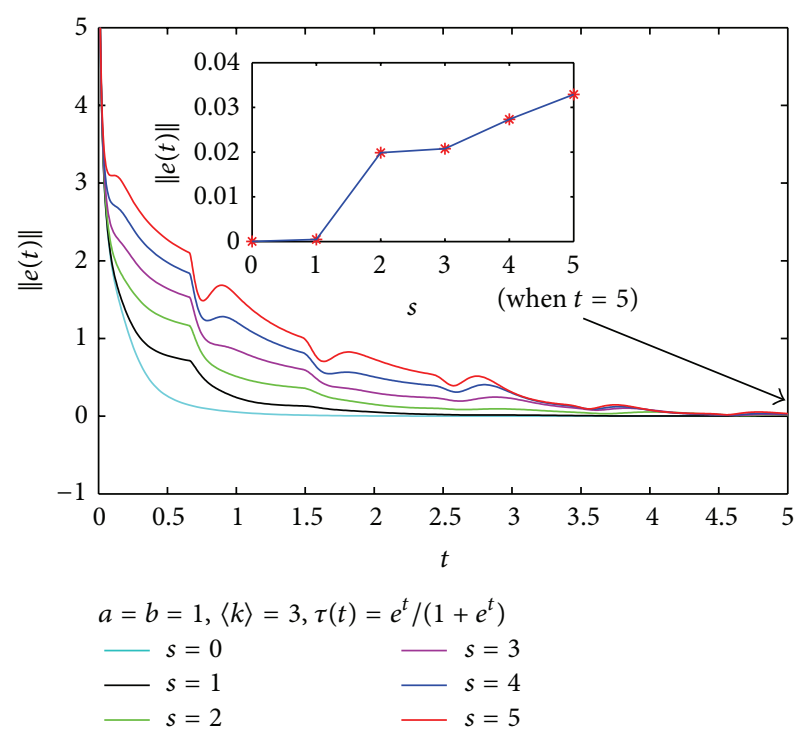

FIgURE 10: The curves of $\|\mathbf{e}(t)\|$ for the networks $A$ and $B$ interlinked following strategy II with $a=b=1,\langle k\rangle=3$, and $\tau(t)=e^{t} /\left(1+e^{t}\right)$ and different internetwork strength $s$.

derived to guarantee that the proposed interdependent networks model is asymmetrically synchronized. Two numerical examples have been provided to illustrate the feasibility and effectiveness of the theoretical results and to further simulate the effects of internetwork delays, intranetwork and internetwork coupling strength on the mutual controlled synchronizability. In comparison, we find that, under the proposed adaptive controllers, the intranetwork coupling strength enhances the mutual synchronization, while the internetwork coupling delays and coupling strength suppress it. This indicates that the synchronization phenomenon in interdependent networks is different from that in a single

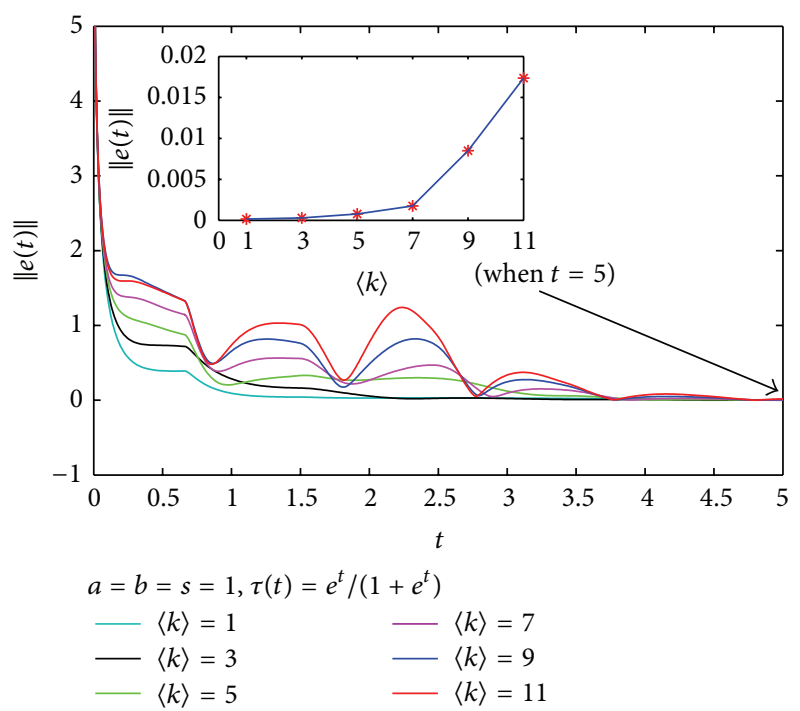

FIgUre 11: The values of $\|e(t)\|$ for the networks $A$ and $B$ interlinked by strategy II with $a=b=s=1, \tau(t)=e^{t} /\left(1+e^{t}\right)$ and different $\langle k\rangle$.

network, which highlights the necessity and significance of considering the mutual synchronization in the context of interdependent networks. Thus, with the help of our findings, one can further understand the mutual synchronization phenomenon in two interdependent networks and design interdependent networks with optimal mutual synchronizability for many potential practical applications.

However, the mutual synchronization between two interdependent networks is extremely complex, and we cannot consider all the factors that influence the synchronizability altogether. Also, our theoretical and numerical results are still conservative and the proposed control schemes are still a bit complicated because of the generality of the model. Therefore, how to simplify the control laws and reduce the number of controlled nodes is another important topic and remains to be researched in future. Thus, utilizing the designed controller, one can derive the synchronization conditions based on Lyapunov function approach, which is widely used in dynamic system analysis and design by some recent articles [40-44].

\section{Conflict of Interests}

The authors declare that there is no conflict of interests regarding the publication of this paper.

\section{Acknowledgments}

This research has been supported by the National Natural Science Foundation of China (Grant no. 51177137) and in part by the China Scholarship Council.

\section{References}

[1] D. J. Watts and S. H. Strogatz, "Collective dynamics of 'smallworld9 networks," Nature, vol. 393, no. 6684, pp. 440-442, 1998. 
[2] S. H. Strogatz, "Exploring complex networks," Nature, vol. 410, no. 6825 , pp. 268-276, 2001.

[3] M. E. J. Newman, "The structure and function of complex networks," Society for Industrial and Applied Mathematics, vol. 45, no. 2, pp. 167-256, 2003.

[4] S. Boccaletti, V. Latora, Y. Moreno, M. Chavez, and D.-U. Hwang, "Complex networks: structure and dynamics," Physics Reports, vol. 424, no. 4-5, pp. 175-308, 2006.

[5] A.-L. Barabási, "Scale-free networks: a decade and beyond," Science, vol. 325, no. 5939, pp. 412-413, 2009.

[6] A. Arenas, A. Díaz-Guilera, J. Kurths, Y. Moreno, and C. Zhou, "Synchronization in complex networks," Physics Reports, vol. 469, no. 3, pp. 93-153, 2008.

[7] M. Zhao, T. Zhou, B.-H. Wang, and W.-X. Wang, "Enhanced synchronizability by structural perturbations," Physical Review E, vol. 72, no. 5, Article ID 057102, 4 pages, 2005.

[8] M. Zhao, T. Zhou, B.-H. Wang, G. Yan, H.-J. Yang, and W.J. Bai, "Relations between average distance, heterogeneity and network synchronizability," Physica A, vol. 371, no. 2, pp. 773780, 2006.

[9] X. F. Wang and G. Chen, "Synchronization in scale-free dynamical networks: robustness and fragility," IEEE Transactions on Circuits and Systems I, vol. 49, no. 1, pp. 54-62, 2002.

[10] B. Liu, X. Liu, G. Chen, and H. Wang, "Robust impulsive synchronization of uncertain dynamical networks," IEEE Transactions on Circuits and Systems I, vol. 52, no. 7, pp. 1431-1441, 2005.

[11] J.-L. Wang and H.-N. Wu, "Local and global exponential output synchronization of complex delayed dynamical networks," Nonlinear Dynamics, vol. 67, no. 1, pp. 497-504, 2012.

[12] Y. Shang, M. Chen, and J. Kurths, "Generalized synchronization of complex networks," Physical Review E, vol. 80, no. 2, Article ID 027201, 4 pages, 2009.

[13] X. Wu and H. Lu, "Projective lag synchronization of the general complex dynamical networks with distinct nodes," Communications in Nonlinear Science and Numerical Simulation, vol. 17, no. 11, pp. 4417-4429, 2012.

[14] M. J. Park, O. M. Kwon, J. H. Park, S. M. Lee, and E. J. Cha, "On synchronization criterion for coupled discrete-time neural networks with interval time-varying delays," Neurocomputing, vol. 99, pp. 188-196, 2013.

[15] N. Mahdavi, M. B. Menhaj, J. Kurths, and J. Lu, "Fuzzy complex dynamical networks and its synchronization," IEEE Transactions on Cybernetics, vol. 43, no. 2, pp. 648-659, 2013.

[16] Z. Li, G. Feng, and D. Hill, "Controlling complex dynamical networks with coupling delays to a desired orbit," Physics Letters A, vol. 359, no. 1, pp. 42-46, 2006.

[17] X. Li, X. Wang, and G. Chen, "Pinning a complex dynamical network to its equilibrium," IEEE Transactions on Circuits and Systems I, vol. 51, no. 10, pp. 2074-2087, 2004.

[18] J. Lu, D. W. C. Ho, J. Cao, and J. Kurths, "Single impulsive controller for globally exponential synchronization of dynamical networks," Nonlinear Analysis, Real World Applications, vol. 14, no. 1, pp. 581-593, 2013.

[19] C. Li, W. Sun, and J. Kurths, "Synchronization between two coupled complex networks," Physical Review E, vol. 76, no. 4, Article ID 046204, 6 pages, 2007.

[20] A. I. Lerescu, N. Constandache, S. Oancea, and I. Grosu, "Collection of master-slave synchronized chaotic systems," Chaos, Solitons and Fractals, vol. 22, no. 3, pp. 599-604, 2004.
[21] H. Tang, L. Chen, J.-A. Lu, and C. K. Tse, "Adaptive synchronization between two complex networks with nonidentical topological structures," Physica A, vol. 387, no. 22, pp. 56235630, 2008.

[22] X. Wu, W. X. Zheng, and J. Zhou, "Generalized outer synchronization between complex dynamical networks," Chaos, vol. 19, no. 1, Article ID 013109, 9 pages, 2009.

[23] C. Jian-Rui, J. Li-Cheng, W. Jian-She, and W. Xiao-Hua, "Adaptive synchronization between two different complex networks with time-varying delay coupling," Chinese Physics Letters, vol. 26, no. 6, Article ID 060505, 4 pages, 2009.

[24] Z. Li and X. Xue, "Outer synchronization of coupled networks using arbitrary coupling strength," Chaos, vol. 20, no. 2, Article ID 023106, 7 pages, 2010.

[25] G. Wang, J. Cao, and J. Lu, "Outer synchronization between two nonidentical networks with circumstance noise," Physica A, vol. 389, no. 7, pp. 1480-1488, 2010.

[26] Q. Bian and H. Yao, "Generalized synchronization between two complex dynamical networks with time-varying delay and nonlinear coupling," Mathematical Problems in Engineering, vol. 2011, Article ID 978612, 15 pages, 2011.

[27] X. Wu and H. Lu, "Outer synchronization of uncertain general complex delayed networks with adaptive coupling," Neurocomputing, vol. 82, pp. 157-166, 2012.

[28] S. Zheng, S. Wang, G. Dong, and Q. Bi, "Adaptive synchronization of two nonlinearly coupled complex dynamical networks with delayed coupling," Communications in Nonlinear Science and Numerical Simulation, vol. 17, no. 1, pp. 284-291, 2012.

[29] Y. Wu, C. Li, Y. Wu, and J. Kurths, "Generalized synchronization between two different complex networks," Communications in Nonlinear Science and Numerical Simulation, vol. 17, no. 1, pp. 349-355, 2012.

[30] S. V. Buldyrev, R. Parshani, G. Paul, H. E. Stanley, and S. Havlin, "Catastrophic cascade of failures in interdependent networks," Nature, vol. 464, no. 7291, pp. 1025-1028, 2010.

[31] S.-W. Mei, Y.-Y. Wang, and L.-J. Chen, "Overviews and prospects of the cyber security of smart grid from the view of complex network theory," High Voltage Engineering, vol. 37, no. 3, pp. 672-679, 2011 (Chinese).

[32] C. D. Brummitt, R. M. D’Souza, and E. A. Leicht, "Suppressing cascades of load in interdependent networks," Proceedings of the National Academy of Sciences of the United States of America, vol. 109, no. 12, pp. E680-E689, 2012.

[33] S. V. Buldyrev, N. W. Shere, and G. A. Cwilich, "Interdependent networks with identical degrees of mutually dependent nodes," Physical Review E, vol. 83, no. 1, Article ID 016112, 8 pages, 2011.

[34] A. Vespignani, "Complex networks: the fragility of interdependency," Nature, vol. 464, no. 7291, pp. 984-985, 2010.

[35] G. Dong, J. Gao, R. Du, L. Tian, H. E. Stanley, and S. Havlin, "Robustness of network of networks under targeted attack," Physical Review E, vol. 87, no. 5, Article ID 052804, 11 pages, 2013.

[36] J. Gao, S. V. Buldyrev, H. E. Stanley, and S. Havlin, "Networks formed from interdependent networks," Nature Physics, vol. 8, no. 1, pp. 40-48, 2012.

[37] J. Shao, S. V. Buldyrev, S. Havlin, and H. E. Stanley, "Cascade of failures in coupled network systems with multiple supportdependence relations," Physical Review E, vol. 83, no. 3, Article ID 036116, 9 pages, 2011.

[38] J. Um, P. Minnhagen, and B. J. Kim, "Synchronization in interdependent networks," Chaos, vol. 21, no. 2, Article ID 025106, 7 pages, 2011. 
[39] H. Zhao, Y. Ma, S. Liu, and Y. Yue, "Fuzzy sliding mode variable structure control of chaotic power system with uncertainty," Journal of Computational Information Systems, vol. 7, no. 6, pp. 1959-1966, 2011.

[40] W. Xiang and J. Xiao, "Finite-time stability and stabilisation for switched linear systems," International Journal of Systems Science, vol. 44, no. 2, pp. 384-400, 2013.

[41] W. Xiang and J. Xiao, "Stability analysis and control synthesis of switched impulsive systems," International Journal of Robust and Nonlinear Control, vol. 22, no. 13, pp. 1440-1459, 2012.

[42] W. Xiang, J. Xiao, and L. Han, "Ho control synthesis for shorttime Markovian jump continuous time linear systems," Circuits, Systems, and Signal Processing, vol. 32, no. 6, pp. 2799-2820, 2013.

[43] W. Xiang, J. Xiao, and M. N. Iqbal, "Ho control for switched fuzzy systems via dynamic output feedback: hybrid and switched approaches," Communications in Nonlinear Science and Numerical Simulation, vol. 18, no. 6, pp. 1499-1514, 2013.

[44] W. Xiang and J. Xiao, "Ho control synthesis of switched discrete-time fuzzy systems via hybrid approach, Optimal Control," Applications and Methods, vol. 34, no. 6, pp. 635-655, 2013. 


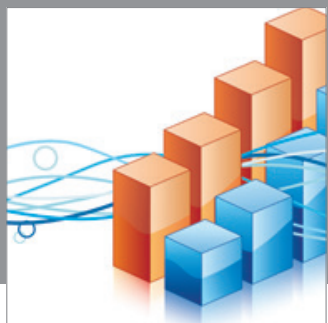

Advances in

Operations Research

mansans

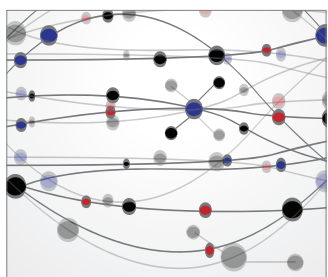

The Scientific World Journal
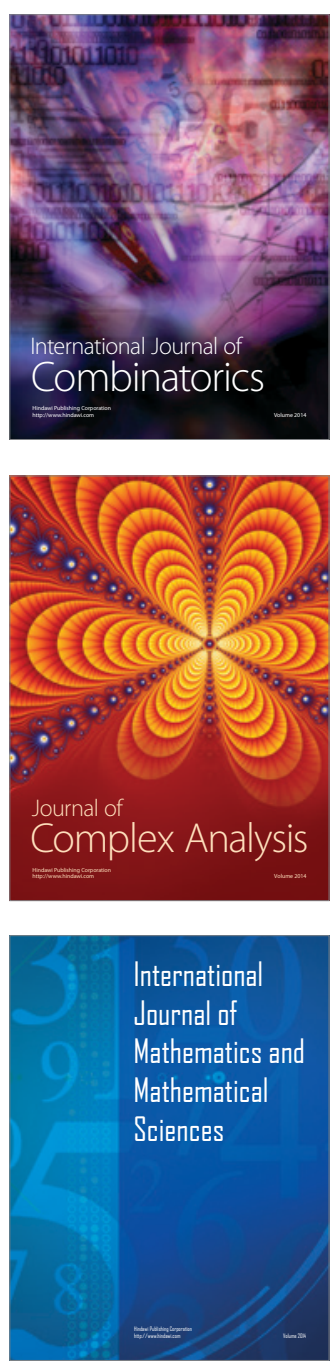
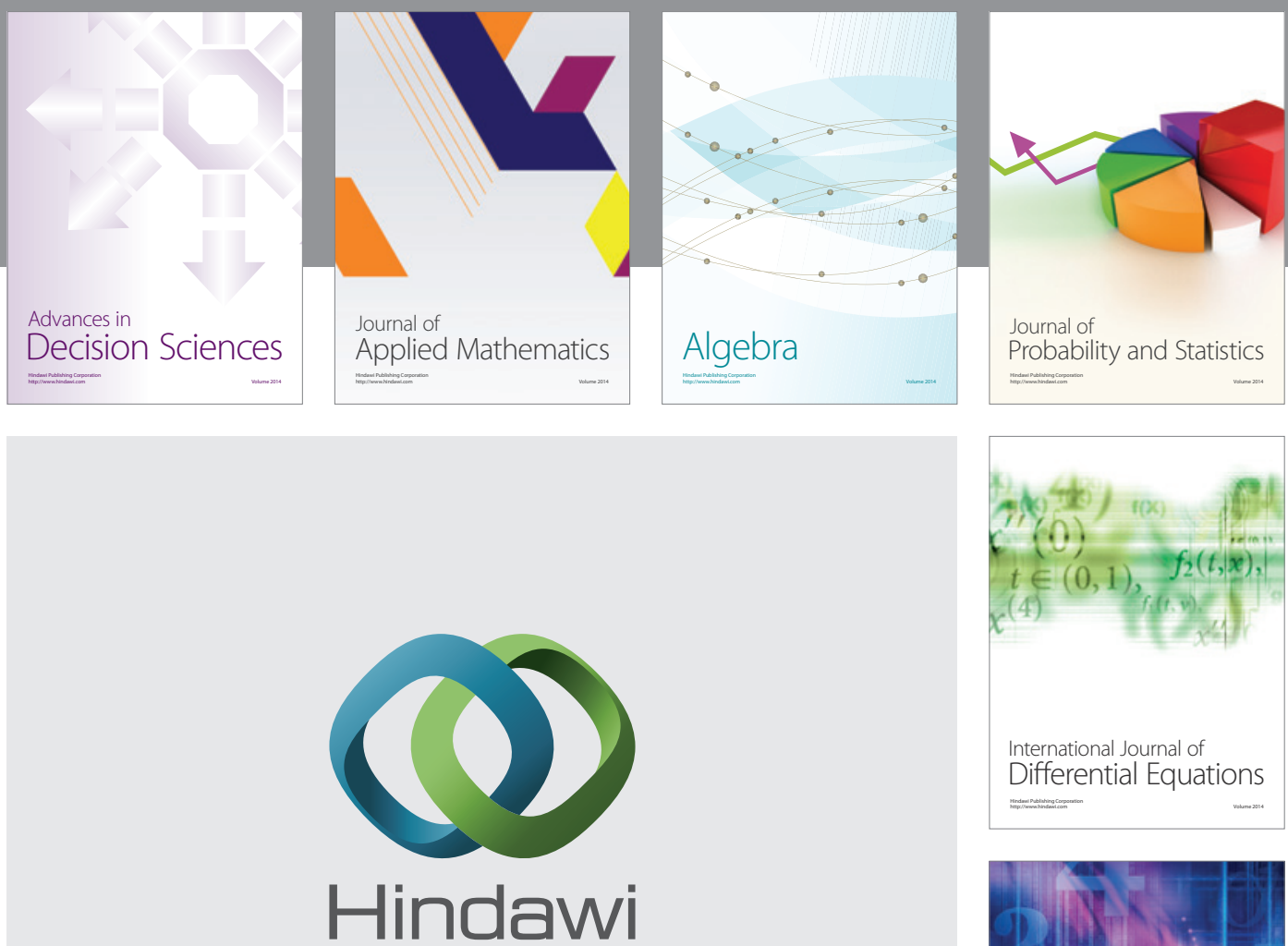

Submit your manuscripts at http://www.hindawi.com
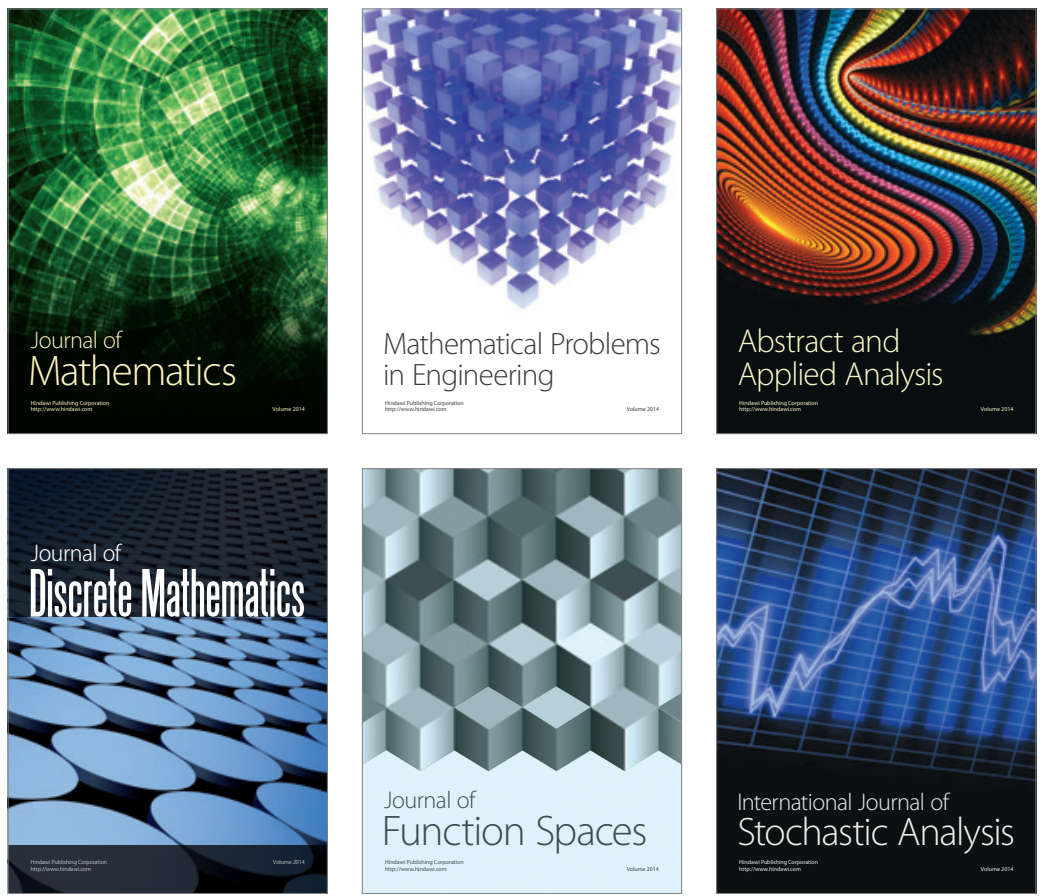

Journal of

Function Spaces

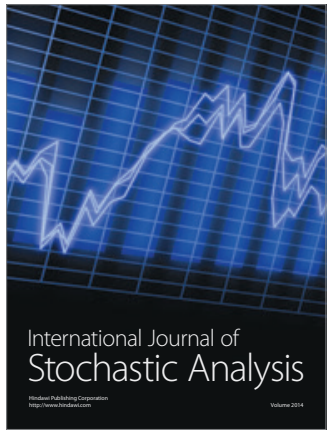

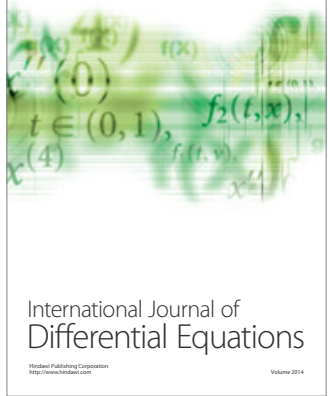
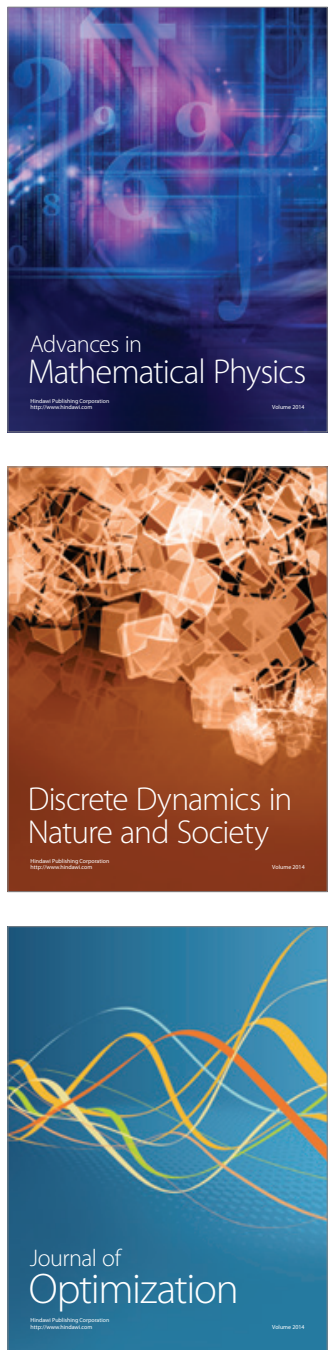